\title{
Aptidão física relacionada à saúde de adolescentes participantes de programa esportivo
}

CDD. 20.ed. 796.07

796.073

\author{
Julia Aparecida Devide NOGUEIRA* \\ Cleilton Holanda PEREIRA*
}

*Faculdade de Educação Física, Universidade de Brasília.

\section{Resumo}

0 presente estudo analisa a aptidão física relacionada à saúde (AFRS) dos adolescentes participantes das Vilas Olímpicas (VO) de Fortaleza, Ceará de acordo com sexo, faixa etária e tempo de participação no programa. É um estudo transversal analítico com amostra representativa e aleatória de 344 adolescentes de ambos os sexos, com idade entre 11 e 16 anos. A massa e estatura corporal foram medidas para cálculo do índice de massa corporal (IMC). Para avaliar AFRS foram medidas flexibilidade, força/resistência muscular (FRM) e aptidão cardiorrespiratória $(\mathrm{ApC})$ utilizando o protocolo do Projeto Esporte Brasil (PROESP-BR). A análise estatística descritiva foi utilizada, seguida de teste t de student, ANOVA "one-way" e regressão logistica binária. Dos participantes, $15,1 \%$ não atingiu os critérios mínimos para IMC; $28,2 \%$ para flexibilidade; $32,6 \%$ para FRM e $45,6 \%$ para $\mathrm{ApC}$, com influência significativa do sexo e faixa etária. Meninos apresentaram maior dificuldade em atender os critérios de flexibilidade $\left(\chi^{2}=5,96 ; p<0,01\right)$ e FRM $\left(\chi^{2}=12,33 ; p<0,001\right)$; e o grupo mais velho apresentou maior dificuldade em atender os critérios para FRM $\left(\chi^{2}=5,74 ; p<0,05\right)$. Adolescentes que participam há mais tempo das V0 tendem a apresentar, de forma não significativa, maiores chances de atingir niveis desejados para flexibilidade ( $R C=1,23$; IC95\%: 0,65-2,33) e $A p C(R C=1,35$; IC95\%: 0,77-2,36) e menores chances para IMC (RC = 0,43; IC95\%: 0,17-1,08) que os que participam há menos tempo. Adolescentes participantes das V0 apresentam elevada prevalência de inaptidão física com algumas diferenças significativas por sexo e faixa etária, mas sem diferença significativa por tempo de participação no programa. Estes resultados indicam a necessidade de reflexão, principalmente por parte dos profissionais da educação física, a respeito das estratégias adotadas nos projetos esportivos para otimizar a promoção de melhoras efetivas na AFRS da população.

PalavRas-Chave: Adolescência; Atividade física; Aptidão cardiorrespiratória; Força e resistência muscular; Flexibilidade; Saúde.

\section{Introdução}

A prevalência e incidência de sedentarismo, obesidade e de doenças crônicas não transmissíveis (DCNT) relacionadas à estes fatores de risco tem aumentado drasticamente nas populaçóes contemporâneas ${ }^{1}$, inclusive entre crianças e adolescentes no Brasil $^{2}$. Alguns relatórios de agências internacionais apontam para o fato de que o nível de atividade física do indivíduo apresenta associação com alguns componentes da aptidáo física como, por exemplo, com a composição corporal, aptidão cardiorrespiratória (ApC) e força/resistência muscular (FRM); e estes, por sua vez, com o estado geral de saúde ${ }^{3-4}$.

Estudos internacionais recentes mostram que a aptidão física relacionada à saúde (AFRS) de grande parte das crianças e adolescentes são insuficientes para uma boa saúde segundo o protocolo e os pontos de corte do Fitnessgram $^{5-7}$. Estudo que aplicou o em 4621 escolares americanos do ensino médio mostrou que $45 \%$ não atingiu a zona de aptidão para $\mathrm{o} \mathrm{IMC}, 19,1 \%$ não atingiu para FRM e 29,6\% não atingiu para $\mathrm{ApC}^{5}$. Estudo com 1206 escolares portugueses de 11 a 15 anos mostrou que $30,7 \%$ estavam acima do peso e $53,3 \%$ deles não atingiam as recomendaçóes para $\mathrm{ApC}^{6}$. Um estudo com 5983 escolares britânicos com idades entre 10 a 16 anos mostrou que $26 \%$ estavam com o IMC acima do peso ideal e $21 \%$ não atingiam as recomendaçôes para $\mathrm{ApC}^{7}$.

Apesar de diferentes protocolos náo permitirem uma comparação direta dos resultados, diversos 
estudos nacionais que utilizaram os critérios do Projeto Esporte Brasil (PROESP-BR) também apontam para a baixa aptidão física de crianças e adolescentes Brasileiros. Estudo epidemiológico transversal com 7507 escolares, $54,8 \%$ do sexo masculino (M) e 45,2\% do feminino (F), de sete a 10 anos de idade, encontrou elevadas prevalências de inaptidão para flexibilidade $(\mathrm{M}=58,3 \%$ e $\mathrm{F}=$ $51,2 \%)$; para FRM (M = 75,3\% e F $=73,8 \%)$; e para ApC $(\mathrm{M}=80,8 \%$ e $\mathrm{F}=77,6 \%) 8$. Estudo com 69 escolares $(\mathrm{M}=50,7 \%)$ de sete a 11 anos de uma escola em Brasília identificou que $17,4 \%$ não foram considerados aptos no IMC; 47,8\% na flexibilidade; e 34,8\% na FRM9. Estudo com 3145 escolares (M $=50,6 \%$ ) de São Paulo entre sete e 16 anos observou prevalência elevada, em geral superior a $50 \%$, para a classificação "ruim" (abaixo do percentil 40) em todos os testes para ambos os sexos, principalmente para as meninas ${ }^{10}$. Estudo com 988 escolares entre sete a 15 anos em São Paulo, indicou que F = $13,2 \%$ e $\mathrm{M}=15,3 \%$ receberam critério fraco para flexibilidade; $\mathrm{F}=27,2 \%$ e $\mathrm{M}=25,7 \%$ para $\mathrm{FRM}$; e $\mathrm{F}=20,8 \%$ e $\mathrm{M}=21,6 \%$ para $A p C^{11}$. Por fim, um estudo que utilizou o protocolo do Fitnessgram em 2849 escolares $(M=48,9 \%)$ de Minas Gerais com idades entre seis e 18 anos identificou que $\mathrm{F}=$ $63,4 \%$ e $\mathrm{M}=72,3 \%$ não atendem aos critérios de saúde no teste de flexibilidade de "sentar e alcançar",

\section{Método}

O presente estudo é aplicado, descritivo e analítico, e foi aprovado pelo Comitê de Ética em Pesquisa da Universidade de Brasília (registro ${ }^{\circ}$ 036/11) e autorizado pela Secretaria de Esportes do CE. O termo de consentimento livre e esclarecido (TCLE) para participação na pesquisa foi assinado pelo adolescente e por um responsável legal.

\section{População e amostra}

Uma amostra representativa da população foi obtida através da lista de alunos matriculados nas $\mathrm{VO}$ no ano de 2011 com idade entre 11 a 16 anos $(n=1421)$. $O$ cálculo do tamanho da amostra aleatória simples utilizou a variância da distribuição por sexo para definir o número amostral (n), considerando-se uma margem de erro aceitável de $5 \%$. Os cálculos de representatividade resultaram em 325 adolescentes. Prevendo a mortalidade amostral, 400 adolescentes foram sorteados para
$\mathrm{F}=23,8 \%$ e $\mathrm{M}=31,2 \%$ não atendem ao de $\mathrm{FRM}$ "abdominal modificado"12.

Considerando que a infância e adolescência representam o período mais importante para o estabelecimento de padróes comportamentais e para o desenvolvimento corporal, cognitivo e motor, intervençôes que busquem aumentar a AFRS nesse período podem contribuir decisivamente para a promoção da saúde em todas as etapas da vida ${ }^{13-15}$. Neste sentido, várias políticas e programas que estimulam a atividade física regular, em geral sob a forma de esportes, têm sido implantados e desenvolvidos no Brasil, principalmente com foco em grupos de risco social como crianças e adolescentes de baixa renda $^{16-19}$. Um destes programas é a Vila Olímpica (VO), que se baseia numa concepção de esporte educacional e inclusáo social, e tem como objetivos melhorar a aptidão física, qualidade de vida e saúde de seus participantes ${ }^{17}$. Embora consensual quanto à importância social e epidemiológica da atividade física em saúde pública ${ }^{1,3-4}$, pouco se sabe a respeito dos efeitos de programas esportivos comunitários ${ }^{16,20}$, inclusive na AFRS. O presente estudo visa contribuir com esta discussão ao analisar a prevalência de atendimento aos critérios de AFRS e sua associação com o sexo, a faixa etária e com o tempo de participação no programa social esportivo da $\mathrm{VO}$ em adolescentes da cidade de Fortaleza, Ceará (CE). participar. Dos 400 adolescentes convidados, 12 não apresentaram TCLE, 27 foram excluídos por preenchimento inadequado dos questionários, nove por estarem fora da faixa etária (abaixo de 11 anos completos ou acima de 16 anos) e oito desistiram durante as avaliaçóes. A amostra final foi composta de 344 adolescentes (236 do sexo masculino e 108 do sexo feminino).

\section{Procedimentos}

Cada VO foi visitada para identificar a disponibilidade das turmas e dos espaços físicos e os dados foram coletados no horário de aula. Os adolescentes selecionados receberam explicaçóes sobre os procedimentos e foram encaminhados a uma sala para confirmação dos dados pessoais, mensuração da massa e estatura corporais e das variáveis de AFRS de acordo com o protocolo PROESP-BR ${ }^{21}$. Cada um dos testes foi realizado pelo mesmo avaliador 
em todas as crianças. Para medir a massa utilizouse uma balança portátil digital (marca Plenna) com capacidade de $120 \mathrm{~kg}$ e precisão de $0,1 \mathrm{~kg}$ e para a estatura utilizou-se fita métrica não extensível com precisão de $0,1 \mathrm{~cm}$ fixada na parede. Para flexibilidade (teste de sentar e alcançar), utilizou-se uma caixa com régua graduada; o aluno sentado de frente para a base, pernas estendidas e unidas, braços estendidos na vertical e mãos uma sobre a outra; ao sinal, inclinou o corpo para frente alcançando o mais longe com as pontas dos dedos das mãos, sem flexionar os joelhos e sem utilizar insistências; foi registrado o melhor resultado entre duas execuçóes ${ }^{21}$.

No teste de FRM (teste de abdominal em um minuto), o aluno posicionou-se em decúbito dorsal com joelhos flexionados a 90 graus e braços cruzados no tórax; o avaliador fixou os pés do aluno ao solo e contou o número de repetiçóes em um minuto; a flexão do tronco deveria levar os cotovelos até as coxas e retornar a posição inicial. No teste de $\mathrm{ApC}$ (teste de corrida de nove minutos) o perímetro da pista foi medido previamente e anotou-se o número de voltas somados à distância percorrida entre a última volta completa e a localização do aluno ao fim do teste; piques de velocidade e caminhadas deveriam ser evitadas; aos nove minutos o apito sinalizou que os alunos deveriam parar e permanecer onde estavam até a distância ser anotada, em metros ${ }^{21}$.

\section{Resultados}

A amostra final foi composta por 344 adolescentes $(68,6 \%$ meninos e $31,4 \%$ meninas). Os valores médios (DP) das variáveis estudadas separadas por sexo, faixa etária e tempo de participação nas VO são apresentados na TABELA 1 .

As prevalências de adolescentes que atenderam aos critérios de AFRS por sexo, faixa etária e tempo de participação nas VO são apresentadas na TABELA 2.

Meninos apresentaram maior dificuldade em atender os critérios de flexibilidade $\left(\chi^{2}=5,96 ; \mathrm{p}<\right.$ $0,01$; ajuste residual $=2,4)$ e FRM $\left(\chi^{2}=12,33 ; \mathrm{p}<\right.$ $0,001$; ajuste residual $=3,5)$ que meninas; o grupo

\section{Análise do dados}

Os dados foram analisados em três recortes: por sexo; faixa etária (11 a 13 anos e 14 a 16 anos); e tempo de participação no programa ( $<1$ ano; $\geq 1 \mathrm{e}<3$ anos; e $\geq 3$ anos). As faixas etárias foram definidas para minimizar diferenças latentes que ocorrem na adolescência e o tempo de participação no programa levou em conta possíveis efeitos fisiológicos adaptativos de curto, médio e longo prazo. Os critérios de saúde para AFRS consideraram os pontos de corte do PROESP-BR ${ }^{21}$.

O teste de Kolmogorov Smirnov foi utilizado para verificar a normalidade dos dados e a análise descritiva utilizou média, desvio padrão (DP) e prevalência (\%). Diferenças entre sexos e faixas etárias foram determinadas pelo teste t de "Student" para amostras independentes. Diferenças por tempo de participação no programa foram avaliadas pela ANOVA “one-way”, uma vez que seu pressupostos foram atendidos, com "Post Hoc" de Tukey. Para analisar as proporçóes de adolescentes que atenderam os critérios de AFRS foi utilizado o teste do Qui-quadrado $\left(\chi^{2}\right)$. A razão de chances $(\mathrm{RC})$ das variáveis sexo, idade e tempo de participação influírem na classificação da AFRS foi calculada por regressão logística binária, com intervalo de confiança de $95 \%$. O nível de significância adotado foi de $\mathrm{p}<0,05$. As análises foram realizadas no "software" SPSS v.15.0.

de 14 a 16 anos apresentou maior dificuldade em atender os critérios para FRM $\left(\chi^{2}=5,74 ; \mathrm{p}<0,05\right.$; ajuste residual $=2,4)$ que os mais novos; e o grupo com menos de um ano de participação nas $\mathrm{VO}$ apresentou maior facilidade em atender os critérios de saúde para IMC $\left(\chi^{2}=5,69 ; \mathrm{p}<0,05\right.$; ajuste residual $=1,5)$. Sabendo que sexo e idade podem influenciar os resultados da AFRS e que os grupos criados por tempo de participação nas VO apresentam proporçôes diferentes por sexo e faixas etárias, os resultados da regressão logística foram ajustados para estas variáveis (TABELA 3). 
TABELA 1 - Média (DP) da idade, massa, estatura e aptidão física relacionada à saúde de acordo com o sexo, faixa etária e tempo de participação nas Vilas Olímpicas (VO).

DP: desvio Padrão; Masc: masculino;

Fem: feminino;

Estat:: estatura

IMC: índice de massa corporal;

Flex: flexibilidade;

FRM: força/resistência muscular;

ApC: aptidão cardiorrespiratória

rep: repetições;

${ }^{*} \mathrm{p}<0,05$;

${ }^{* *} p<0,01$;

$* * * p<0,001$ pelo tes-

te $t$ de "student" para

amostras independen-

tes entre sexos e entre

faixas etárias, e pela

ANOVA "one-way" com

"post hoc" de Tukey para tempo de participação nas VO.

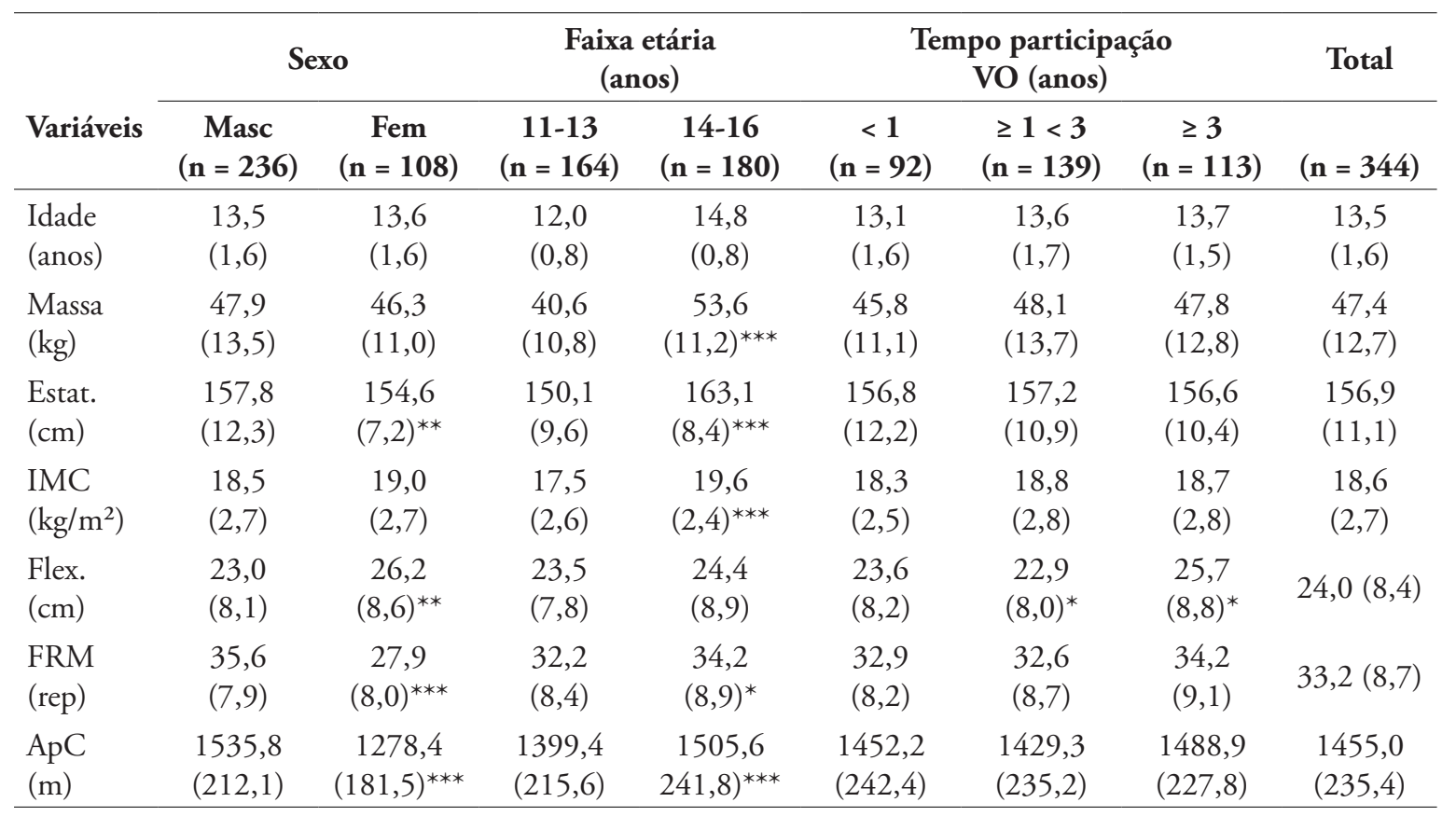

TABELA 2 - Prevalência (\%) de adolescentes que atenderam os critérios do PROESP-BR para aptidão física relacionada à saúde de acordo com o sexo, faixa etária e tempo de participação nas Vilas Olímpicas (VO).

PROESP-BR: Projeto Esporte Brasil;

Masc: masculino;

Fem: feminino;

IMC: índice de massa

corporal;

Flex: flexibilidade;

FRM: força/resistência

muscular;

ApC: aptidão cardiorrespiratória;

${ }^{*} p<0,05$;

${ }^{* *} p<0,01$

${ }^{* * *} p<0,001$ pelo teste do qui-quadrado entre os sexos, as faixas etárias e o tempo de participação nas VO.

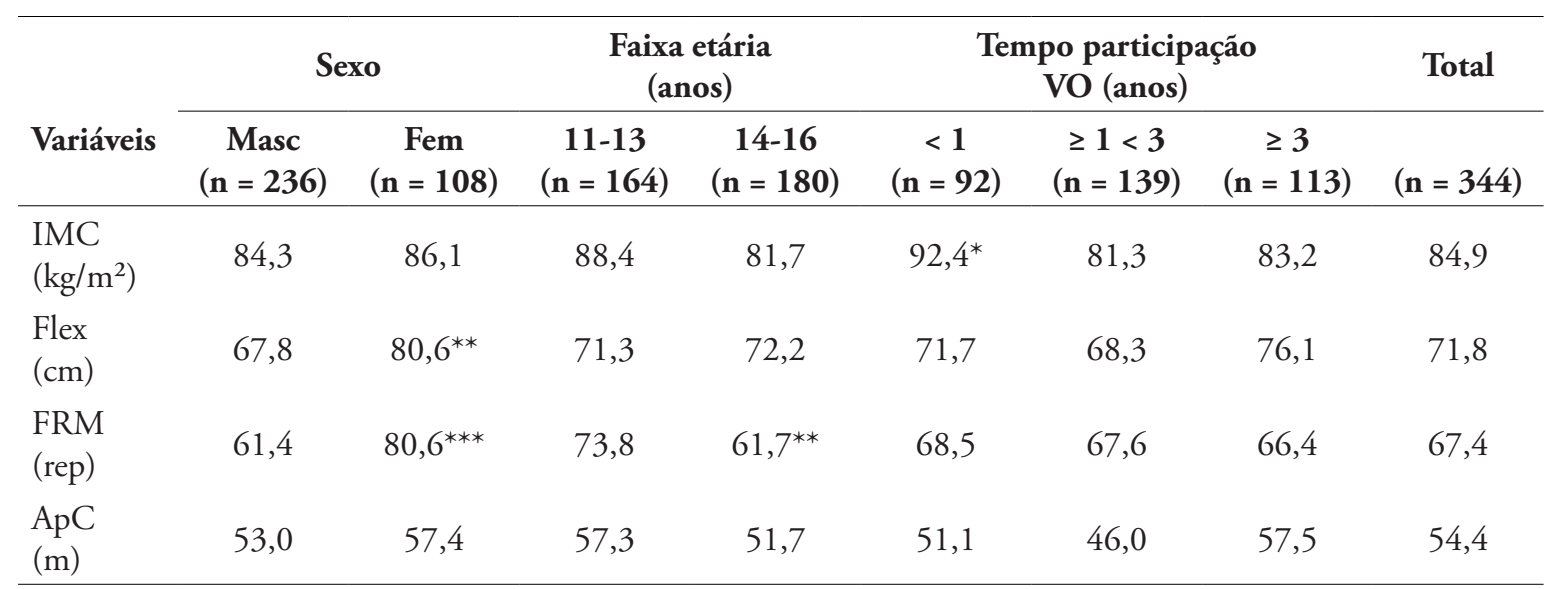

34 • Rev Bras Educ Fís Esporte, (São Paulo) 2014 Jan-Mar; 28(1):31-40 
TABELA 3 - Razão de chance (IC 95\%) para atingir os níveis desejados de aptidão física relacionada à saúde ajustada para sexo, idade e tempo de participação nas Vilas Olímpicas (VO).

\begin{tabular}{|c|c|c|c|c|c|c|c|}
\hline \multirow[b]{2}{*}{ Variáveis } & \multicolumn{2}{|c|}{ Sexo } & \multicolumn{2}{|c|}{ Faixa etária (anos) } & \multicolumn{3}{|c|}{ Tempo participaçáo VO (anos) } \\
\hline & $\begin{array}{c}\text { Masc } \\
(\mathrm{n}=\mathbf{2 3 6}) \\
\end{array}$ & $\begin{array}{c}\text { Fem } \\
(n=108)\end{array}$ & $\begin{array}{c}11-13 \\
(n=164)\end{array}$ & $\begin{array}{c}14-16 \\
(\mathrm{n}=180)\end{array}$ & $\begin{array}{c}<1 \\
(\mathrm{n}=92)\end{array}$ & $\begin{array}{c}\geq 1<3 \\
(\mathrm{n}=139)\end{array}$ & $\begin{array}{c}\geq 3 \\
(\mathbf{n}=113)\end{array}$ \\
\hline $\operatorname{IMC}\left(\mathrm{kg} / \mathrm{m}^{2}\right)$ & 1 & $\begin{array}{c}1,22 \\
(0,63-2,36)\end{array}$ & 1 & $\begin{array}{c}0,63 \\
(0,34-1,18)\end{array}$ & 1 & $\begin{array}{c}0,38 \\
(0,15-0,93)\end{array}$ & $\begin{array}{c}0,43 \\
(0,17-1,08)\end{array}$ \\
\hline Flex $(\mathrm{cm})$ & 1 & $\begin{array}{c}1,97^{*} \\
(1,13-3,43)\end{array}$ & 1 & $\begin{array}{c}0,63 \\
(0,34-1,18)\end{array}$ & 1 & $\begin{array}{c}0,82 \\
(0,45-1,49)\end{array}$ & $\begin{array}{c}1,23 \\
(0,65-2,33)\end{array}$ \\
\hline FRM (rep) & 1 & $\begin{array}{c}2,72^{* *} \\
(1,57-4,72)\end{array}$ & 1 & $\begin{array}{c}0,53^{*} \\
(0,33-0,86)\end{array}$ & 1 & $\begin{array}{c}1,03 \\
(0,57-1,85)\end{array}$ & $\begin{array}{c}0,96 \\
(0,52-1,77)\end{array}$ \\
\hline ApC (m) & 1 & $\begin{array}{c}1,20 \\
(0,76-1,91)\end{array}$ & 1 & $\begin{array}{c}0,76 \\
(0,49-1,18)\end{array}$ & 1 & $\begin{array}{c}1,17 \\
(0,68-2,00)\end{array}$ & $\begin{array}{c}1,35 \\
(0,77-2,36)\end{array}$ \\
\hline
\end{tabular}

IC: intervalo de confiança; Masc: masculino; Fem: feminino; IMC: índice de massa corporal; Flex: flexibilidade; FRM: força/resistência muscular;

ApC: aptidão cardiorrespiratória; ${ }^{*} p<0,05$; ${ }^{* *} p<0,01$;

nível de significância pela análise de regressão logística ajustada por sexo, idade e tempo de participação nas VO.

\section{Discussão}

O programa esportivo das $\mathrm{VO}$ é implantado em áreas de baixo poder aquisitivo, elevado risco social e com estrutura precária de escolas e postos de saúde ${ }^{17}$. Apesar da reconhecida relevância social deste tipo de iniciativa $^{4,16-19}$, pouco se sabe sobre a prevalência de aptidão física nestes grupos populacionais e, segundo o conhecimento dos autores, nada se publicou sobre os efeitos do tempo de participação em programas que promovem atividade física na AFRS. Este é o primeiro estudo a avaliar os efeitos do tempo de participação em um programa esportivo no atendimento de critérios da AFRS em adolescentes brasileiros.

A amostra selecionada foi representativa e indicou numero significativamente maior de meninos participantes, fato corroborado pela literatura ${ }^{22,23}$ uma vez que aspectos psicossociais estimulam que garotos pratiquem mais atividades físicas e que meninas desenvolvam atividades "comportadas" e sedentárias ${ }^{915}$. Entretanto, ao analisar a prevalência de inaptos entre os participantes separados por sexo, este e outros estudos ${ }^{8,11,23}$ indicaram maior prevalências de meninos que náo atenderam aos níveis desejados de AFRS pelo PROESP-BR.

Quando comparadas com outros dois estudos desenvolvidos no Estado do $\mathrm{CE}^{23-24}$, as prevalências de inaptidão física obtidas no presente estudo são menores, com exceção para flexibilidade, quando comparado com o estudo realizado com 755 estudantes participantes e não participantes de programa esportivo na cidade de Fortaleza ${ }^{23}$. Neste, as prevalências de inaptidáo para flexibilidade ocorreram em $21,7 \%$ dos meninos e $11,0 \%$ das meninas; FRM em $69,1 \%$ dos meninos e $63,9 \%$ das meninas; e ApC em 55,6\% dos meninos e $51,5 \%$ das meninas ${ }^{23}$. O outro estudo com 233 escolares de Juazeiro do Norte mostrou as prevalencias de inaptidáo para flexibilidade foram 78,9\% em meninos e 77,8\% em meninas; FRM em $69,0 \%$ dos meninos e $57,1 \%$ das meninas; e ApC em $57,1 \%$ dos meninos e $66,9 \%$ das meninas ${ }^{24}$. Considerando que durante a adolescência meninas em geral são menos ativas ${ }^{9,22}$, que os valores médios para FRM e ApC são maiores em meninos, e que não existem aspectos fisiológicos ou culturais que expliquem maiores prevalências de inaptidão física em meninos, pode-se questionar se os pontos de corte propostos pelos testes de AFRS realmente atendem aos critérios de sensibilidade e especificidade necessários para identificar riscos à saúde em adolescentes ${ }^{25}$.

As diferenças observadas entre os sexos nos valores médios dos componentes da AFRS também podem ter ocorrido devido à alteraçóes decorrentes da puberdade, quando alteraçôes hormonais regulam o crescimento e diferenciam as características físicas: meninos aumentam a massa magra, ficam mais altos e pesados, e meninas aumentam a massa gorda ${ }^{15}$, resultando em melhor desempenho dos meninos, em valores absolutos, nos testes de $\mathrm{FRM}^{9-11,23}$ e de $\mathrm{ApC}^{9,23}$. Já para flexibilidade, diferenças anatômicas 
favorecem as meninas ${ }^{10,15}$. Além disto, questôes culturais podem ampliar o contraste ao reforçar os efeitos da testosterona ${ }^{15}$ na competitividade masculina ${ }^{11}$ e ao estimular açóes motoras intensas para meninos e atividades de flexibilidade para meninas?

Ao analisar os resultados por faixas etárias, observa-se que adolescentes mais velhos apresentam maiores valores médios de AFRS. Tais resultados sáo esperados em decorrência da maturaçáo ${ }^{15}$ mas a flexibilidade tende a diminuir durante o estirão de crescimento devido ao maior comprimento proporcional das pernas em relaçáo à estatura e ao crescimento ósseo longitudinal mais acentuado que músculos e tendôes ${ }^{13,15}$. Entretanto, verificou-se que adolescentes mais velhos apresentam maior prevalência de inaptidão física, com diferença significativa para FRM quando comparados a alunos mais novos. Outros estudos que utilizaram o protocolo do PROESP-BR também identificaram maiores prevalências de inaptos entre alunos mais velhos ${ }^{11,24,26}$.

Estudo com 223 escolares do CE, com idades entre 11 e 16 anos, concluiu que há um declínio da aptidáo física dos adolescentes com o passar da idade, apresentando prevalência de $71,8 \%$ de inaptos para FRM entre alunos mais velhos 24 . Outro estudo com 665 escolares do Rio Grande do Sul, com idade média (DP) de 11,0 (2,0) anos, identificou que o IMC, a FRM e a ApC estiveram associados com características biológicas de sexo e idade ${ }^{26}$. Estudos com escolares com idades entre sete a 15 anos $^{11}$ e seis a 18 anos $^{12}$ mostrou que os componentes da AFRS foram significativamente influenciadas pela idade e sexo, onde o sexo feminino mostrou-se mais propenso à inaptidão ${ }^{11-12}$. Um estudo com 1206 adolescentes portugueses entre 11 a 15 anos mostrou que maiores prevalências de sobrepeso e de baixa ApC estavam relacionadas com o avanço do processo de maturação ${ }^{6}$, mas outro estudo com 1110 crianças portuguesas entre seis e 10 anos concluiu que a idade não parece ser um fator significativo nos níveis de AFRS nesta faixa etária ${ }^{22}$. Cabe destacar que em adolescentes alterações morfofuncionais podem ocorrer em decorrência tanto da atividade física quanto do estirâo de crescimento e da maturaçáo sexual ${ }^{15}$. Neste sentido, após ajustes para essas variáveis de confusão, o presente estudo mostrou que a $\mathrm{RC}$ em atingir níveis desejados de AFRS para meninas foi maior para flexibilidade e FRM; e adolescentes mais novos apresentaram RC significativamente maior de atingir níveis desejados para FRM.

Em relação ao tempo de participação nas $\mathrm{VO}$, alunos com mais de três anos apresentaram resultados médios significativamente maiores para flexibilidade e discreta tendência para maior FRM e ApC que os grupos com menos tempo de participação, mas não houve diferenças significativas nas prevalências de atendimento aos critérios de AFRS. Ademais, os adolescentes com mais de três anos nas VO tendem a apresentar chances maiores, embora não significativas, em atingir níveis desejados de flexibilidade e ApC. Por outro lado, tendem a apresentar menores chances, não significativas, em atingir níveis desejados de IMC e FRM. Não foram encontrados outros estudos que investigam a relaçáo entre tempo de participação e AFRS em adolescentes para comparação.

Alguns estudos apontam que a participação regular em ao menos 60 minutos de atividades físicas moderada a intensa pode contribuir para a melhoria da saúde de crianças e jovens através de benefícios psicológicos, morfológicos, funcionais e metabólicos ${ }^{4,7,27}$, mas outros estudos que comparam resultados da AFRS em participantes e náo participantes de programas esportivos sugerem relações fracas $^{23}$, moderadas $^{28}$ ou até inexistentes ${ }^{22}$. Estudo com 755 adolescentes de 10 a 14 anos não identificou diferenças significativas nas médias das variáveis de AFRS entre os que participavam e os que não participavam do projeto esportivo, mas mostrou que adolescentes participantes do projeto social esportivo apresentam chances (IC 95\%) significativamente maiores em alcançar níveis desejados de flexibilidade $[R C=1,45(0,98-2,14) ; \mathrm{p}=0,05]$, FRM [RC $=1,48(1,09-2,01) ; \mathrm{p}=0,01]$ e $\mathrm{ApC}[\mathrm{RC}=1,42$ $(1,06-1,89) ; \mathrm{p}=0,01]$ que os não ativos ${ }^{23}$. Estudo experimental de intervenção com 383 escolares de 10 a 15 anos mostrou melhores resultados para FRM e ApC no grupo participante de atividades físicas por um ano comparado ao grupo não participante, mas não houve diferenças significativas no momento pós-intervenção comparado ao pré-intervenção ${ }^{28}$.

Estudo com 4621 estudantes do ensino médio dos Estados Unidos mostrou que aqueles que náo cumprem as recomendaçôes mínimas de atividade física têm maiores chances de não atingir níveis saudáveis de ApC, IMC e FRM, e que o aumento no número de dias de atividades aeróbias foi acompanhada de diminuiçáo da RC de ser inapto 5 . Outro estudo com 5983 escolares entre 10 a 16 anos da Inglaterra mostrou que aumentar maiores níveis de $\mathrm{ApC}$ resultavam em menor pressão arterial média em adolescentes obesos de ambos os sexos ${ }^{7}$. Estudo de revisão que utilizou a metodologia de metaanálise, sobre aptidão física e atividade física como fatores de risco separados para doenças cardíacas, 
concluiu que ser inapto é um fator de risco distinto de ser inativo; e que a formulação de recomendaçôes de atividade física com base em estudos de aptidão podem rebaixar a importância da aptidão física como um fator de risco, enquanto exagerar os benefícios de quantidades moderadas de atividade física para a saúde ${ }^{29}$.

Um estudo realizado apenas com adolescentes que praticam atividade física regular em clubes ou escolas esportivas há mais de um ano e participam das aulas de educação física escolar em Londrina - Paraná, mostrou que $41 \%$ deles náo atenderam aos critérios do protocolo do PROESP-BR para o IMC, 19\% pata FRM e $45 \%$ para $\mathrm{ApC}^{30}$. A intensidade, duração e frequência em que a atividade física é realizada são fundamentais para a obtenção de benefícios fisiológicos à saúde e à aptidão física ${ }^{4,29}$, e a intensidade pode exercer maior influência em alguns componentes da AFRS que a quantidade total de atividade ${ }^{31}$.

É importante destacar que o presente estudo apresenta algumas limitaçóes, como a não mensuração do volume e intensidade da atividade física; da frequência de participação nas aulas e do nível de motivação dos alunos; aspectos relevantes na determinação do nível de atividade física e de seus efeitos na AFRS. Entretanto, os projetos sociais em geral assumem um limite máximo de faltas de $25 \%$ para que a criança possa continuar inscrita no programa; uma característica que, de certa forma, minimiza o problema da ausência de controle da frequência. Não obstante a tais limites, os resultados encontrados são corroborados por outros estudos que apontam níveis de aptidão física insuficientes para uma boa saúde em crianças e adolescentes ${ }^{8-12,24}$, inclusive entre os considerados fisicamente ativos ${ }^{22-23,28,30}$.

Além disto, este estudo contribui de forma inédita na discussáo sobre os efeitos da permanência em programas esportivos comunitários na AFRS de crianças e adolescentes. É possível que após o período inicial de adaptações às atividades físicas, estas não promovam melhoras adicionais na AFRS por não representarem uma sobrecarga ao estado atual do indivíduo. Como o programa da $\mathrm{VO}$ se baseia na concepção de esporte educacional, é possível que haja um predomínio do jogo e de atividades lúdicas, que acabam por privilegiar as capacidades motoras coordenativas em detrimento as capacidades motoras condicionais, tais como FRM e resistência cardiorrespiratória $^{32}$. Por outro lado, verifica-se que atividades de maior intensidade podem estar relacionadas à menor adesão em programas de exercícios físicos ${ }^{33}$, gerando a necessidade de mais estudos sobre os efeitos da adesão, motivação e quantidade/intensidade da atividade física na AFRS e no estado geral de saúde de adolescentes brasileiros.

É ainda fundamental que se institua uma cultura avaliativa nos programas sociais esportivos ${ }^{20}$, produzindo continuamente dados sobre os efeitos obtidos na AFRS e, caso necessário, que se reoriente suas intervenções para melhorar a eficácia, pois programas de atividade física e esporte tem sido amplamente divulgados e difundidos socialmente como alternati-

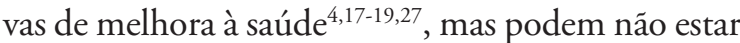
resultando nos níveis de aptidão física necessários para promover a saúde. Estes são alguns aspectos que merecem atenção de pesquisadores e profissionais da área de saúde, em especial os professores de educação física, envolvidos com programas e políticas públicas de saúde, educação e esporte que visam ampliar o acesso à atividade física e promover a saúde de crianças e adolescentes no Brasil.

A participação de crianças e adolescentes do sexo feminino no programa das VO é significativamente menor que a de meninos, e estas apresentam resultados médios significativamente menores para FRM e ApC, indicando a necessidade de professores e gestores repensarem as atividades desenvolvidas e estratégias de captação e manutenção da adesão a partir das diferenças entre os sexos e faixas etárias.

Destaca-se ainda que uma parcela significativa dos adolescentes de ambos os sexos não atingiram os critérios mínimos para AFRS, especialmente para ApC; e que o tempo de permanência no projeto esportivo da $\mathrm{VO}$ não aumentou significativamente as chances de obter níveis desejados de AFRS. Estes resultados sugerem que as atividades físicas desenvolvidas no programa das $\mathrm{VO}$ não sejam suficientes para promover níveis de AFRS necessários à prevenção de DCNT em seus participantes.

Tais resultados podem subsidiar gestores, coordenadores e profissionais da saúde envolvidos com atividade física e esporte, em especial os professores de educação física, a refletir sobre as finalidades e os métodos propostos de forma que a dinâmica de execução dos projetos sociais possa efetivamente atingir seus objetivos e promover a saúde da populaçáo. 


\section{Abstract \\ Health related fitness of adolescents participating in a sports program}

The present study aims to analyze AFRS of adolescent participating in the Olympic Villages (VO) in Fortaleza, Ceará according to sex, age and permanence in the program. This is an analytical cross-sectional study with random representative sample of 344 adolescents of both sexes, aged between 11 and 16 years. Body weight and height were measured to calculate BMI. To evaluate AFRS, flexibility, muscular strength/endurance (FRM) and cardiorespiratory fitness (ApC) were measured using the Esporte Brasil (PROESP-BR) protocol. For data analysis, descriptive statistics, Student's t test, one-way ANOVA and binary logistic regression were used. Of the participants, $15.1 \%$ did not meet the minimum criteria for BMI; $28.2 \%$ for flexibility, $32.6 \%$ for FRM and $45.6 \%$ for $\mathrm{ApC}$, with significant influence of sex and age. Boys were more difficult to meet the criteria for flexibility $\left(\chi^{2}=5.96, p<0.01\right)$ and FRM $\left(\chi^{2}=12.33\right.$, $p<0.001)$, and the older group showed greater difficulty in meeting the criteria for FRM $\left(\chi^{2}=5.74, p\right.$ $<0.05)$. Teens who participate longest at the $\mathrm{VO}$ tend to be, more likely to achieve desired levels for flexibility (not significantly) $(\mathrm{OR}=1.23,95 \% \mathrm{Cl}: 0.65-2.33)$ and $\mathrm{ApC}(\mathrm{RC}=1.35,95 \%$ : $0.77-2.36)$ and fewer chances for $\mathrm{BMI}(\mathrm{OR}=0.43,95 \% \mathrm{Cl}: 0.17-1.08)$ than those involved more recently. Adolescent participants in $\mathrm{VO}$ have a high prevalence of physical low fitness, especially ApC, with significant influence of sex and age, but unrelated to the permanence in the program. These results indicate the need for reflection, especially by physical education professionals, about the strategies adopted in the sports projects to effectively promote improvements in AFRS of the population.

KEY wORDS: Adolescence; Physical activity; Cardiorespiratory fitness; Muscular strength and endurance; Flexibility; Health.

\section{Referências}

1. WHO. World Health Organization. Noncommunicable diseases country profiles 2011. WHO Global Report. 2011.

2. Brasil. IBGE. Instituto Brasileiro de Geografia e Estatística Pesquisa Nacional de Saúde do Escolar (PeNSE) 2012. Rio de Janeiro: IBGE; 2013.

3. O'Donovan G, Blazevich AJ, Boreham C, et al. The abc of physical activity for health: a consensus statement from the British Association of Sport and Exercise Sciences. J Sports Sci. 2010;28:573-91.

4. Haskell WL, Lee I, Pate RR, et al. Physical activity and public health: updated recommendation for adults from the American College of Sports Medicine and the American Heart Association. Med Sci Sports Exerc. 2007;39:1423-34.

5. Morrow JR, Tucker JS, Jackson AW, Martin SB, Greenleaf CA, Petrie TA. Meeting physical activity guidelines and health-related fitness in youth. Am J Prev Med. 2013;44:439-44.

6. Coelho-e-Silva MJ, Ronque ERV, Cyrino ES, et al. Nutritional status, biological maturation and cardiorespiratory fitness in Azorean youth aged 11-15 years. BMC Public Health. 2013;13:495-505.

7. Ogunleye AA, Sandercock GR, Voss C, Eisenmann JC, Reed K. Prevalence of elevated mean arterial pressure and how fitness moderates its association with BMI in youth. Public Health Nut. 2012;1:1-9.

8. Pelegrini A, Augusto D, Silva S, Estadual U. Aptidão física relacionada à saúde de escolares brasileiros: dados do Projeto Esporte Brasil. Rev Bras Med Esporte. 2011;17:92-6.

9. Pereira CH, Ferreira DS, Copetti GL, et al. Aptidão física em escolares de uma unidade de ensino da rede pública de Brasília-DF. Rev Bras Ativ Fís Saúde. 2011;16:223-7.

10. Luguetti CN, Tereza M, Böhme S. Indicadores de aptidão física de escolares da região centro-oeste da cidade de São Paulo. Rev Bras Cineantropom Desempenho Hum. 2010;12:331-7.

11. Andreasi V, Michelin E, Rinaldi AEM, Burini RC. Physical fitness and associations with anthropometric measurements in 7 to 15-year-old school children. J Pediatr. 2010;86:497-502.

12. Guedes DP, Miranda NJT, Germano JM, Lopes V, Silva AJRM. Aptidão física relacionada à saúde de escolares: programa fitnessgram. Rev Bras Med Esporte. 2012;18:72-6. 
13. Mikkelsson L, Kaprio J, Kautiainen H, Kujala U. School fitness tests as predictors of adult health-related fitness. Am J Hum Biol. 2006;18:342-9.

14. Ortega FB, Ruiz JR, Castillo MJ. Physical activity, physical fitness, and overweight in children and adolescents: evidence from epidemiologic studies. Endocrinol y Nutr. 2013; 60:458-69.

15. Rogol AD, Clark PA, Roemmich JN. Growth and pubertal development in children and adolescents: effects of diet and physical activity. Am J Clin Nutr. 2000;72:S521-8.

16. Vianna JA, Lovisolo HR. Projetos de inclusão social através do esporte: notas sobre a avaliação. Mov. 2009;15:145-62.

17. Ceará. Secretaria do Esporte. Vila Olímpica: Projeto Pedagógico. 2008. [citado 20 fev. 2013]. Disponível em: http:// www.esporte.ce.gov.br/index.php/vilas-olimpicas.

18. Brasil. Ministério da Educação e da Saúde. Programa Saúde na Escola. 2007. Decreto no: 6.286. (5 dezembro de 2007). [ citado 15 fev. 2013]. Disponível em: http://www.planalto.gov.br/ccivil_03/_Ato2007-2010/2007/Decreto/D6286.htm.

19. Brasil. Ministério do Esporte. Secretaria de Esporte Educacional. Programa Segundo Tempo. 2003. [citado 20 fev. 2013]. Disponível em: http://portal.esporte.gov.br/snee/segundotempo/default.jsp.

20. U.S. Department of Health and Human Services. Physical activity evaluation handbook. Atlanta: U.S. Department of Health and Human Services, Centers for Disease Control and Prevention; 2002.

21. Gaya A, Silva G. PROESP-BR: observatório permanente dos indicadores de saúde e fatores de prestação esportiva em crianças e jovens - manual de aplicação de medidas e testes, normas e critérios de avaliação. Porto Alegre: UFRGS/ CNPq/Ministério do Esporte; 2007.

22. Santos DMV, Chaves RN, Souza MC, Seabra A, Garganta R. Taxas de sucesso na aptidão física: efeitos da idade, sexo, atividade física, sobrepeso e obesidade. Rev Bras Cineantropom Desempenho Hum. 2010;12:309-15.

23. Souza EA. Associação da prática de atividade física com a aptidão física relacionada à saúde em escolares da cidade de fortaleza [dissertação]. Brasília: Universidade de Brasília; 2010.

24. Costa CLA, Banceira PFR, Nobre GC, Nobre FSS. Aptidão física relacionada à saúde em escolares de Juazeiro do Norte. Mov Percepc. 2010;11:48-56.

25. Paludo AC, Batista MB, Serassuelo Júnior H, Ronque ERV. Aptidáo cardiorrespiratória em adolescentes estimada pelo teste de corrida e/ou caminhada de 9 minutos. Rev Bras Cineantropom Desempenho Hum. 2012;14:401-8.

26. Dumith SC, Azevedo Júnior MR, Rombaldi AJ. Aptidão física relacionada à saúde de alunos do ensino fundamental do município de Rio Grande, RS, Brasil. Rev Bras Med Esporte. 2008;14:454-9.

27. WHO. World Health Organization. Global recommendations on physical activity for health. Geneva: WHO; 2010.

28. Farias ES, Carvalho WRG. Efeito da atividade física programada sobre a aptidão física em escolares adolescentes. Rev Bras Cineantropom Desempenho Hum. 2010;12:98-105.

29. Williams PT. Physical fitness and activity as separate heart disease risk factors: a meta-analysis. Med Sci Sports Exerc. 2001;33:754-61.

30. Schubert A, Januário RSB, Casonatto J, Sonoo CN. Imagem corporal, estado nutricional, força de resistência abdominal e aptidão cardiorrespiratória de crianças e adolescentes praticantes de esportes. Rev Paul Pediatr. 2013;31:71-6.

31. Ruiz JR, Rizzo NS, Hurtig-Wennlöf A, Ortega FB, Wärnberg J, Sjöström M. Relations of total physical activity and intensity to fitness and fatness in children: the European Youth Heart Study. Am J Clin Nutr. 2006;84:299-303.

32. Farinatti P, Ferreira M. Saúde, promoção da saúde e educação física. Rio de Janeiro: UERJ; 2006.

33. Perri MG, Anton SD, Durning PE, et al. Adherence to exercise prescriptions: effects of prescribing moderate versus higher levels of intensity and frequency. Health Psychol. 2002;21:452-8.

\section{Agradecimentos}

Agradecemos pelo apoio financeiro da Coordenação de Aperfeiçoamento de Pessoal de Nível Superior (CAPES), na forma de bolsa de mestrado, ao autor Cleilton Holanda Pereira. 
Cleilton Holanc Caculdade de Educaça Pereira Universidade de Brasília Campus Universitário Darcy Ribeiro - Asa Norte 70910-970 - Brasília - DF - BRASIL e-mail: cleilton.holanda@gmail.com
Recebido para publicação: 11/03/2013

Revisado: 24/10/2013

Aceito: 16/01/2014 